\title{
Complicaciones del abordaje anterior en la patología de la columna cervical
}

R. García-Armengol; S. Colet-Esquerre; P. Teixidor-Rodríguez; M. Alamar-Abril; J.M. Cladellas-Ponsa; C. HostalotPanisello; J. Muñoz-Aguiar y R. Florensa-Brichs

Servicio de Neurocirugía. Hospital Universitari Germans Trias i Pujol. Badalona. Barcelona.

Resumen

Objetivo. Analizar las complicaciones de pacientes intervenidos mediante abordaje cervical anterior en la patología de la columna.

Material y métodos. Estudio retrospectivo de una serie de 193 casos clínicos, entre Diciembre de 1989 y Diciembre de 2004, en el Hospital Germans Trias i Pujol de Badalona, donde se analizaron las complicaciones surgidas con el abordaje cervical anterior y su relación con las distintas técnicas aplicadas. Se analizaron variables sociodemográficas (edad, sexo), variables clínicas (sintomatología inicial, origen de la patología cervical, tipo de complicación, y tiempo de duración de la complicación) y quirúrgicas (número de niveles intervenidos, nivel intervenido, tipo de intervención realizada, tipo de injerto utilizado). A partir de los datos obtenidos se realizó un análisis estadístico con modelos de análisis multivariante, con la prueba de T-Student y con el test de Chi-cuadrado para analizar la relación entre las complicaciones y las distintas variables estudiadas.

Resultados. De todos los individuos estudiados (193), hallamos complicaciones en 50 pacientes (25,91\%). De ellas, la más frecuente fue la disfagia, presente en 15 pacientes. La mayoría de ellas se presentaron de forma transitoria (13 pacientes) y en muy pocas ocasiones de forma permanente ( 2 pacientes). El análisis estadístico mediante la prueba de la $\mathbf{T}$ de Student mostró que no existían diferencias estadísticamente significativas $(p=0,431)$ entre las edades de los pacientes que habían presentado complicaciones frente a los que no, y tampoco se encontraron diferencias estadísticamente significativas, utilizado el test de la Chi-cuadrado, respecto el sexo $(p=0,515)$, síntomas iniciales $(p=0,923)$, origen de la patología $(p=0,364)$, tipo de intervención realizada $(p=0,295)$, y tipo de injerto utilizado $(p=0,382$,). Donde sí encontramos diferencias estadísticamente significativas fue en el número de niveles intervenidos $(p=0,018)$ con una razón de las ventajas para el número de niveles

Recibido: 3-04-06. Aceptado: 22-08-06 (único/múltiple) de 2,221. Con el análisis multivariante siguiendo en modelo de regresión lineal considerando edad, sexo y número de niveles intervenidos, observamos que persistía el riesgo de complicaciones del número de espacios intervenidos, independientemente de la edad o el sexo, siendo los múltiples espacios un $117,3 \%$ más frecuente que la intervención de un único (OR 2,173; IC95\% 1,104-4,279)

Conclusiones. 1. La cirugía de la columna cervical por vía anterior, es una técnica simple, y un procedimiento quirúrgicamente seguro con un número bajo de complicaciones. 2. La disfagia es la complicación más frecuente, pero que se encuentra casi inherente al procedimiento y en la mayoría de ocasiones se resuelve sin tratamiento. 3. Han sido muchos los procedimientos utilizados para la fijación de la columna cervical, con más de 40 años de experiencia, $y$ aún queda por definir cual es el mejor. Harían falta más estudios de carácter multicéntrico y de cohorte prospectiva para poder comparar resultados clínicos, radiológicos, y la presencia de complicaciones.

Anterior approach complications in cervical spine pathology

Summary

Objective. To analyze cervical discectomy complications with anterior approach.

Material and methods. Retrospectively clinical cases series of 193 patients, between December 1989 to December 2004, in the Hospital Germans Trias i Pujol in Badalona. We analyzed complications arisen with cervical anterior approach and its relation with differents procedures. Demographic variables (age, sex), clinical variables (onset symptoms, cervical pathology cause, complication type, and duration time) and surgical variables (operated levels number, procedure, graft used) were analyzed. We made a statistical analysis with multivariant analysis, T-Student test and Chi-square test to analyze the relation between the complications 
and different studied variables.

Results. We found complications in $\mathbf{5 0}$ patients $(25,91 \%)$. Most frequent has been dysphagia, present in 15 patients. Most of them appear with transitory form (13 patients) and in very few occasions permanent form (2 patients). T-Student test in statistical analysis showed no statistically significant differences $(p=0,431)$ between the ages and complications, and were not statistically significant differences, used Chi-square test, in sex $(p=0,515)$, in onset symptoms $(p=0,923)$, in pathology origin $(p=0,364)$, in a procedure $(p=0,295)$, and graft used $(p=0,382)$. We found statistically significant differences in operated levels number $(p=0,018)$ with a ratio for the advantages (single /multiple) of 2,221. Multivariant analysis with linear regression model considering age, sex and operated levels number, showed that persistist the risk of complications in operated number levels, independently of the age or sex, being the multiple spaces a $117.3 \%$ more frequent than the single space (OR 2,173; IC95\% 1,104-4,279).

Conclusions. 1. Cervical anterior approach, is a simple technique, and safe surgically procedure with under number of complications. 2. Dysphagia is most frequent complication, but it is inherent to the procedure and it is solved without treatment in most ocasions. 3. Have been many the procedures used for the cervical spine fixation, with more than 40 years of experience, and still it is to define as he is the best one. It would be necessary more studies of prospective multicentric character to be able to compare clinical, radiological results, and the presence of complications.

\section{Introducción}

Las tres técnicas más conocidas de abordajes anteriores de la columna cervical, fueron descritas por Cloward ${ }^{13}$, Smith \& Robinson ${ }^{70}$, y Bayley \& Badley ${ }^{7}$. Todas ellas permiten el acceso directo tanto a los cuerpos vertebrales como a los discos intervertebrales, además de la artrodesis con la aplicación de un injerto óseo. La interposición de injerto lleva consigo por ello, la posible expulsión, colapso, o reabsorción del mismo con o sin el desarrollo de cifosis, impactación en la médula espinal o pseudoartrosis ${ }^{23,50}$. Años después, Orozco-Delclos y Llovet-Tapies ${ }^{51}$, desarrollaron la utilización de la placa metálica y los tornillos somáticos que con diferentes modificaciones se mantienen en la actualidad mejorando los resultados quirúrgicos, añadiendo estabilidad y previniendo la migración del injerto. Posteriormente se desarrollaron diferentes sistemas de placas con tornillos unicorticales. Su aplicación mejoró los resultados quirúrgicos, con menores aflojamientos, roturas de los tornillos o extrusiones de las placas ${ }^{20}$, sin embargo, también fueron publicadas un número no despreciable de complicaciones desde su utilización, incluyendo desplazamientos del sistema, de los tornillos, o deformación de los mismos $^{50}$. Paralelamente se popularizó la utilización del injerto bovino a fin de minimizar las complicaciones (dolor en el sitio donante) que se producía con el injerto autólogo, que fue substituido rápidamente por el injerto heterólogo debido a las complicaciones publicadas ${ }^{56,63}$. Otra solución a este problema fueron las cajas intersomáticas de diferentes aleaciones (titanio, peek, etc...) que añadían estabilidad, manteniendo la altura del espacio intervertebral ${ }^{27,40,52}$. En los últimos 5 años, se han empezado a utilizar las prótesis de disco, las cuales han añadido además, de la restauración de la curva lordótica del raquis cervical, una mayor preservación de su función, así como la disminución del estrés biomecánico en los segmentos adyacentes ${ }^{69}$.

El objetivo del presente trabajo es el estudio retrospectivo de las complicaciones posquirúrgicas de las discectomías cervicales anteriores en 193 pacientes intervenidos en el Hospital Germans Trias i Pujol, en los últimos quince años, y analizar dichas complicaciones para determinar una posible relación con las diferentes variables sociodemográficas y clínicas estudiadas.

\section{Material y métodos}

Estudio retrospectivo de una serie de 193 casos clínicos (entre Diciembre de 1989 y Diciembre del 2004) en el Hospital Germans Trías i Pujol de Badalona, donde se analizaron las complicaciones surgidas con el abordaje cervical anterior y su relación con las distintas técnicas utilizadas. Se excluyeron del estudio aquellos pacientes cuyo seguimiento era inferior a los dos años o sus datos eran incompletos en su historia clínica. La fuente de información primaria fue la base de datos del servicio de Neurocirugía del Hospital Germans Trías i Pujol de Badalona.

Se realizó un abordaje anterolateral derecho tipo Smith-Robinson ${ }^{70}$ en todos los casos, practicado por dos neurocirujanos del Servicio (R. F. y S. C.). Se colocó un drenaje redón postoperatorio y se realizó profilaxis antibiótica.

En todos los casos se practicó radiología simple preoperatoria de columna cervical en proyecciones anteroposterior y perfil. Desde 1989 hasta 1992, se completó el estudio con tomografía computarizada (TC) de la columna cervical, y a partir del año 1992 se introdujo la resonancia magnética (RM) como exploración complementaria.

La electromiografía se utilizó en la mayoría de casos como complemento al diagnóstico.

Se analizaron variables sociodemográficas (edad, sexo), variables clínicas (sintomatología inicial, tipo de la patología cervical, tipo de complicación, y tiempo de duración de la complicación), y variables quirúrgicas (número de niveles intervenidos, nivel intervenido, tipo de intervención 
realizada, tipo de injerto utilizado).

Primeramente se procedió a un análisis descriptivo de la muestra por medio de media y desviación estándar para variables cuantitativas y por medio de porcentajes para variables cualitativas. Posteriormente se estudió su distribución según el test de Kolmogorov-Smirnov ( $p>0,05)$, y se estudió la asociación entre variables cuantitativas y la presencia o no de complicaciones por medio del test de T-Student. Para evaluar la asociación estadísticamente significativa entre variables cualitativas y presencia o ausencia de complicaciones se utilizó el test de Chi-cuadrado o Fisher según convino.

Aquellas variables explicativas que presentaban una asociación estadísticamente significativa $(\mathrm{p}<0,05)$ con la variable resultado o dependiente (complicaciones) y no presentaban interacciones entre ellas fueron introducidas en un modelo de regresión logística para evaluar su capacidad predictiva de manera independiente.

\section{Variables clínicas}

\section{Sintomatología inicial}

La sintomatología inicial se agrupó en tres tipos. En el primero se incluyeron pacientes con afectación radicular, en el segundo pacientes con signos de mielopatía, y en el tercer grupo pacientes con afectación mielorradicular.

\section{Tipo de la patología cervical}

Se analizó la causa por la cual fueron intervenidos los pacientes incluidos en el estudio y se distinguieron tres grupos. En uno de ellos se incluyó a los pacientes que presentaba patología discal, en otro grupo los que presentaba patología espondilótica, y finalmente un tercer grupo compuesto por pacientes que presentaban patología de origen traumático, y patología tumoral.

\section{Características de las complicaciones}

Se clasificaron en tres grupos, el primero incluyó complicaciones relacionadas con el abordaje y procedimiento quirúrgico, tales como complicaciones laríngeas (disfonías, afonías), traqueales, esofágicas (rotura, laceración, molestias deglutorias) y faríngeas, la lesión de los nervios laríngeos, la rotura de grandes vasos, la alteración de la cadena simpática-cervical, el aumento del déficit neurológico (radicular, medular) como consecuencia directa de la intervención, la laceración dural o la fístula del líquido cefalorraquídeo, los hematomas de partes blandas y los problemas respiratorios derivados de éstos, la infección superficial o profunda (discitis, meningitis, absceso). En el segundo grupo se incluyeron las complicaciones derivadas del material de artrodesis, tanto del injerto (extrusión, colapso, fractura), como de la instrumentación (fractura de la placa, rotura, extrusión de los tornillos, extrusión de la caja intersomática). Las complicaciones sistémicas constituyeron el tercer grupo.

\section{Duración de la complicación}

Establecimos como complicación transitoria aquélla que no perduraba pasados los tres meses, y como complicación permanente, aquélla que se mantenía pasado este tiempo.

\section{Variables quirúrgicas}

\section{Número de niveles intervenidos}

Se establecieron dos grupos. En el primero se incluyeron aquellos pacientes intervenidos de un único nivel y en el segundo grupo los pacientes intervenidos de dos o más niveles.

\section{Tipo de intervención}

La indicación de cada procedimiento se determinó en función del tipo de patología, la presencia de inestabilidad, la experiencia del cirujano, así como la época en que se realizó la intervención, ya que se fueron abandonando métodos a medida que se fueron incorporando otros, a lo largo de los quince años.

Los procedimientos mayoritariamente utilizados fueron las discectomías simples, las discectomías con injerto de diferentes tipos con y sin placa atornillada, las cajas intersomáticas y las corporectomías. En los dos últimos años se ha empezado a emplear la artroplastia discal, utilizada preferentemente en pacientes jóvenes ( $<50$ años).

\section{Tipo de injerto}

Básicamente se utilizaron tres tipos de injerto. El injerto autólogo (hueso esponjoso de cresta ilíaca), el xenoinjerto (hueso esponjoso bovino), y el injerto heterólogo constituido de hidroxiapatita o de fosfato tricálcico.

Los datos fueron analizados con el programa SPSS para Windows versión 12.0 (SPSS Inc, 2001).

\section{Resultados}

\section{Variables sociodemográficas}

De toda la población estudiada $(n=193), 121(63 \%)$ eran hombres y 72 (37\%) mujeres (Figura 1), con un rango de edad entre los 29 a 75 años, y una media de 48,8 años (+/-11,5 años). (Figura 2 )

En cuanto a las variables clínicas, 98 (50,8\%) fueron intervenidos por patología discal, 77 (39,9\%) por patología espondilótica, y $18(9,3 \%)$ por patología traumática o patología tumoral (Figura 3). La clínica inicial, en 106 pacientes $(54,9 \%)$ fue de radiculopatía, en $80(41,45 \%)$ de mielopatía, y en $7(3,63 \%)$ de mielorradiculopatía. De todos los pacientes estudiados, 141 (73,1\%) fueron 


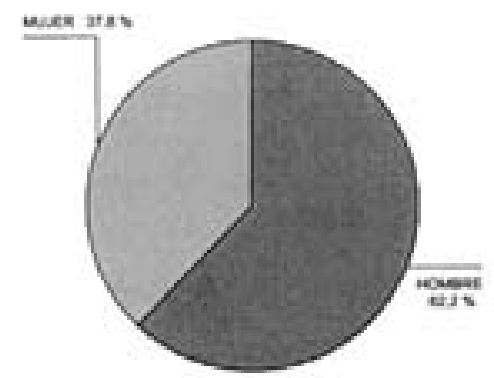

Figura 1. Distribución de sexo

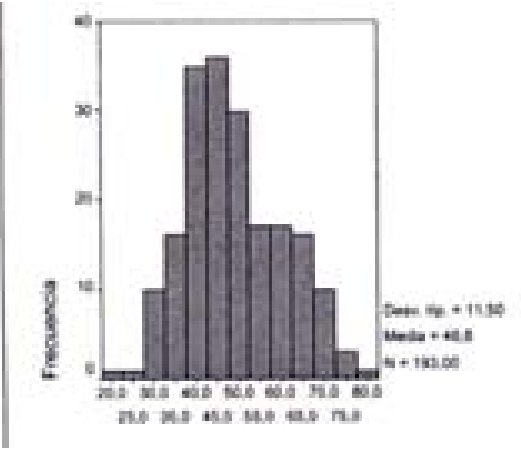

Figura 2. Distribución de edades

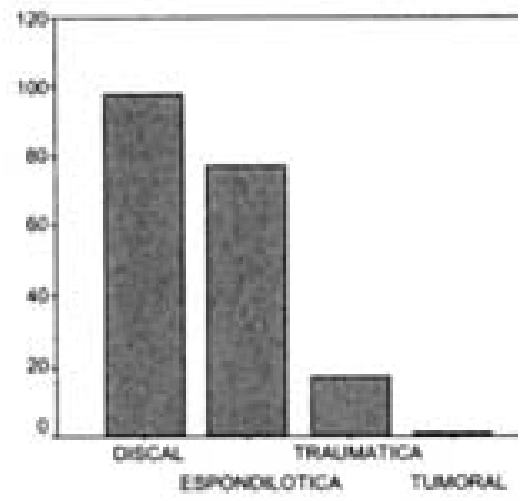

Figura 3. Origen de la patología

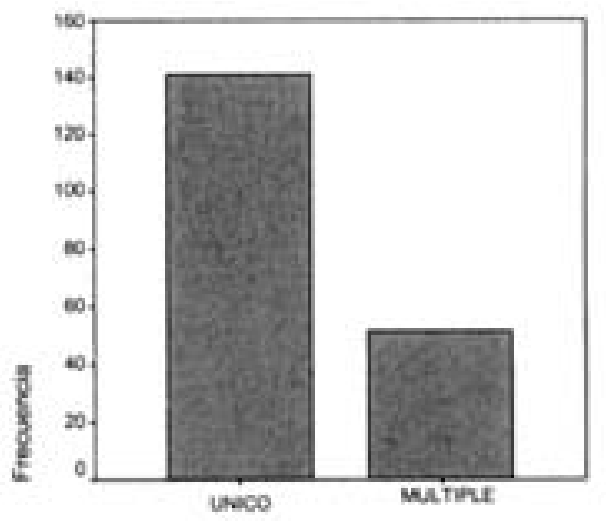

Figura 4. Numero de niveles intervenidos intervenidos de un único nivel, mientras que $52(26,9 \%)$ fueron intervenidos de múltiples niveles (Figura 4) El tipo de intervención realizada, se resume en la tabla 1 , siendo la discectomía y artrodesis con placa e injerto la intervención quirúrgica practicada con mayor frecuencia (87 pacientes $(45 \%))$.

\section{Complicaciones}

De los 193 pacientes estudiados, 50 presentaron algún tipo de complicación. El tipo de complicaciones queda resumido en la tabla 2.

De las complicaciones surgidas a consecuencia del procedimiento quirúrgico, la más frecuente fue la disfagia con 15 pacientes, todas ellas transitorias, con una duración máxima de 3 meses. La disfonía apareció en 7 pacientes, en 5 fueron disfonías transitorias, y en 2 casos se mantuvieron de forma permanente. En 5 casos se produjo por edema de partes blandas (edema de cuerdas vocales), todas ellas transitorias, 1 por luxación de cartílagos laríngeos y 1 caso por lesión del nervio laríngeo recurrente. Un caso de hematoma posquirúrgico que se diagnosticó a las 48 horas de la intervención quirúrgica y a las 24 horas de la retirada del drenaje redón. Precisó intubación inmediata por insuficiencia respiratoria aguda, con buena evolución posterior. En 4 pacientes se objetivó una lesión radicular nueva (en 3 casos se resolvió de forma transitoria precoz y en 1 se mantuvo de forma permanente). En 4 pacientes se objetivó clínicamente una lesión medular nueva. En todos ellos se trató de una complicación transitoria precoz, que

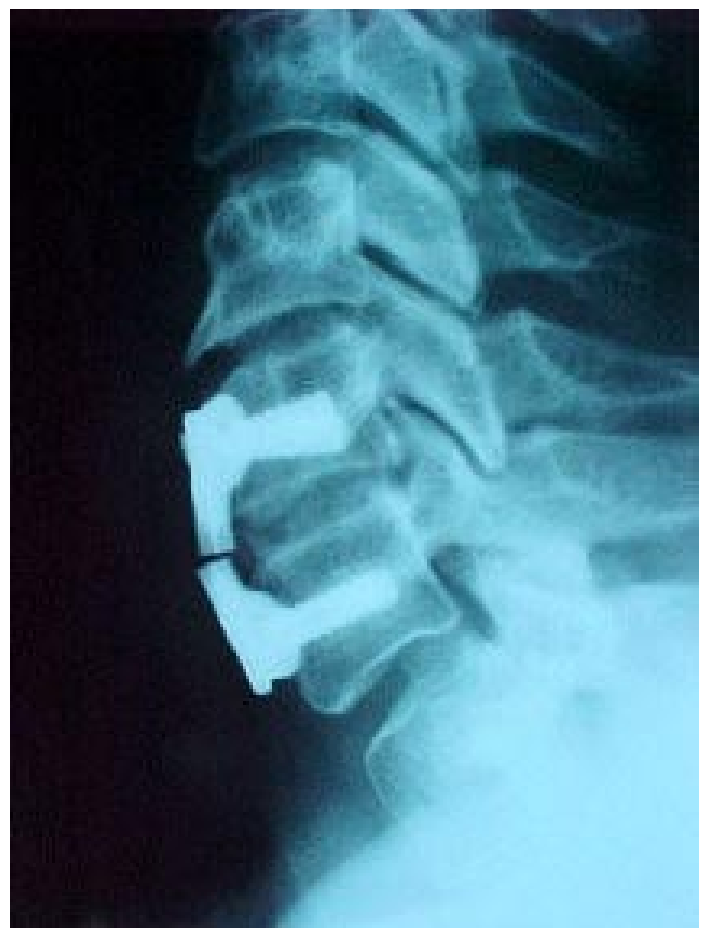

Figura 5. Rotura de una placa. 
Tabla1

Tipo de intervención

\begin{tabular}{|l|c|c|c|c|}
\hline & Frecuencia & $\%$ & $\%$ válido & \% acumulado \\
\hline Discectomía simple & 9 & 4,7 & 4,7 & 4,7 \\
Discectomía + injerto & 17 & 8,8 & 8,8 & 13,5 \\
Discectomía + caja & 69 & 35,8 & 35,8 & 49,2 \\
Discectomía + placa + injerto & 84 & 43,5 & 43,5 & 92,7 \\
Discectomía + caja + placa & 2 & 1,0 & 1,0 & 93,8 \\
Corporectomía + artrodesis & 4 & 2,1 & 2,1 & 95,9 \\
Prótesis de disco & 8 & 4,1 & 4,1 & 100,0 \\
Total & 193 & 100,0 & 100,0 & \\
\hline
\end{tabular}

no perduró más allá de los tres meses, y en la mayoría de los casos se resolvió durante la primera semana. En un caso se produjo una infección superficial de la herida quirúrgica (frotis cutáneo positivo para Escherichia Coli) que se resolvió con tratamiento antibiótico durante 14 días, con buena cicatrización posterior.

De las complicaciones relacionadas con el injerto, se produjo 1 caso de extrusión del injerto óseo autólogo que precisó reintervención. El colapso del injerto se constató en 3 casos, todos ellos injertos bovinos, asintomático en 1 de ellos, un caso precisó tratamiento quirúrgico, por compresión esofágica de un pequeño fragmento, y un caso intervención con abordaje foraminal por reducción de espacio y compromiso radicular.

En cuanto a las complicaciones relacionadas con el material de osteosíntesis, 4 casos de rotura de una placa

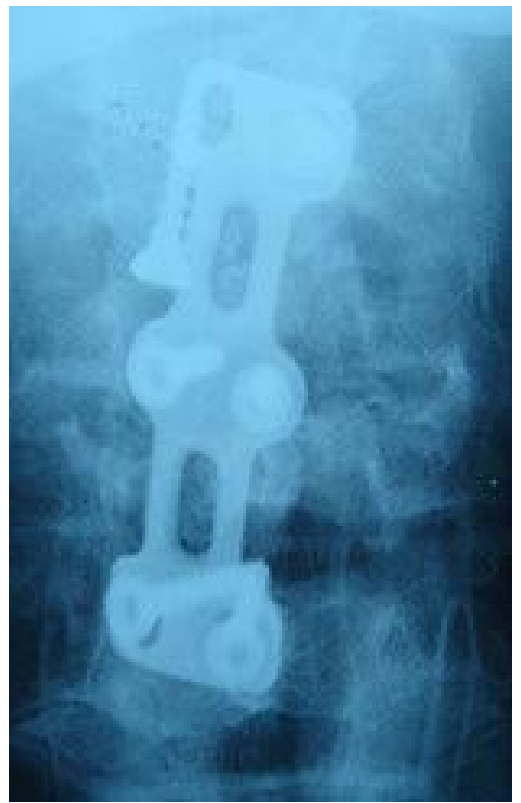

Figura 6. Extrusión de un tornillo (fig. 5), 3 de las cuales precisaron reintervención, y en un caso se detectó en un control a los 5 años, clínicamente asintomática. Se produjeron extrusiones de un tornillo (fig. 6) (tornillos sin seguro de cierre) en 4 pacientes, todos tratados de forma quirúrgica, en dos de ellos por clínica de disfagia. Un caso de fractura de un tornillo, sin clínica acompañante, ni reintervención posterior necesaria. En solo un caso de los tratados con cajas intersomáticas se apreció extrusión de la misma. (Figura 7).

Las complicaciones sistémicas consistieron en dos

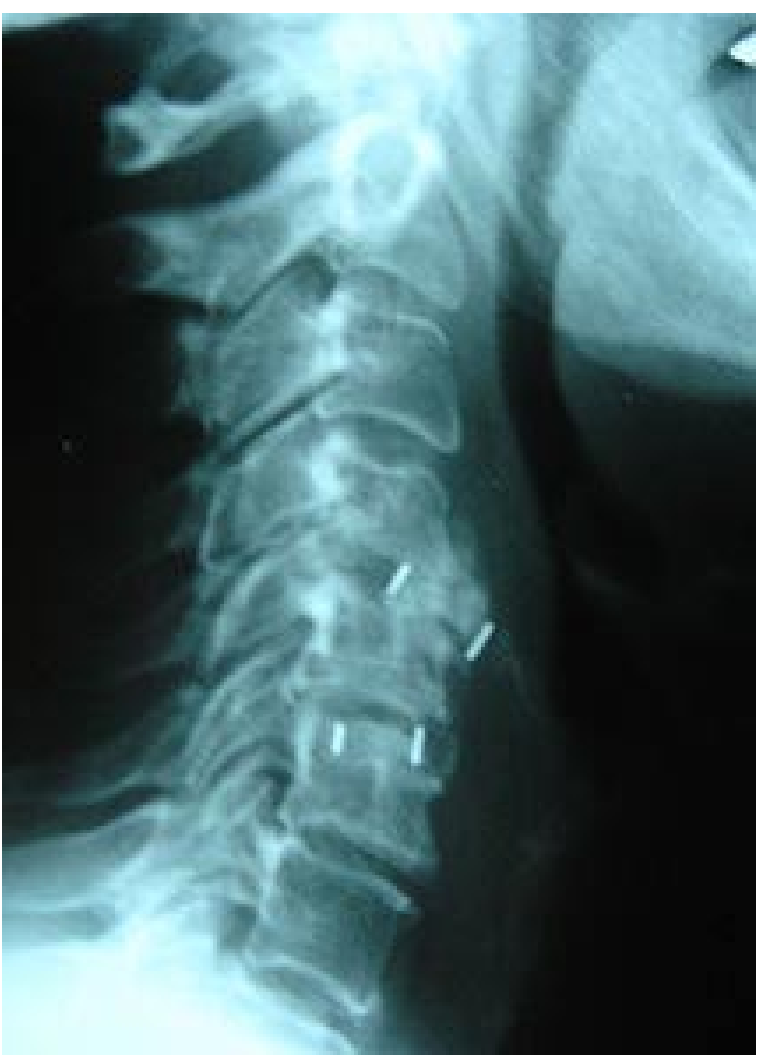

Figura 7. Extrusión de una caja intersomática 
Tabla 2

Complicaciones (50 complicaciones en 193 pacientes)

\begin{tabular}{|l|c|c|c|c|}
\hline \multicolumn{7}{|c|}{ Tipo de complicación } \\
\hline & Frecuencia & $\%$ & Transitoria & Permanente \\
\hline Abordaje/Procedimiento quirúrgico & & & & \\
\hline Disfagia & 15 & 7,7 & 13 & 2 \\
\hline Disfonía & 7 & 3,63 & 5 & 2 \\
\hline Alteración medular & 4 & 2,07 & 4 & - \\
\hline Alteración radicular & 4 & 2,07 & 3 & 1 \\
\hline Hematoma partes blandas & 1 & 0,52 & 1 & - \\
\hline Infección herida quirúrgica & 1 & 0,52 & 1 & - \\
\hline Complicaciones sistémicas & & & & \\
\hline - Infección orina & 2 & 1,03 & 2 & - \\
\hline - Infección respiratoria & 2 & 1,03 & 2 & - \\
\hline Complicaciones osteosintesis & & & & \\
\hline Complicaciones del injerto & 4 & 2,07 & 2 & 2 \\
\hline - Extrusión & 1 & 0,52 & 1 & - \\
\hline - Colapso & 3 & 1,55 & 1 & 2 \\
\hline Complicaciones instrumentación & 10 & 5,18 & 5 & 5 \\
\hline - Fractura placa & 4 & 2,07 & 3 & 1 \\
\hline - Extrusión tornillo & 4 & 2,07 & 1 & 3 \\
\hline - Fractura tornillo & 1 & 0,52 & - & 1 \\
\hline - Extrusión caja intersomática & 1 & 0,52 & 1 & - \\
\hline
\end{tabular}

casos de trombosis venosa profunda, y dos casos de neumonía. No hubo mortalidad en relación a la cirugía en nuestra serie.

El análisis estadístico mediante la prueba de la T de Student mostró que no existían diferencias estadísticamente significativas $(\mathrm{p}=0,431)$ entre las edades de pacientes que habían presentado complicaciones frente a los que no, y tampoco se encontraron diferencias estadísticamente significativas, utilizado el test de la Chi-cuadrado, en el sexo $(\mathrm{p}=0,515)$, en los síntomas iniciales $(\mathrm{p}=0,923)$, en el origen de la patología $(\mathrm{p}=0,364)$, en el tipo de intervención realizada $(p=0,295)$, y en el tipo de injerto utilizado $(p=0,382$,$) .$ Donde sí encontramos diferencias estadísticamente significativas fue en el número de niveles intervenidos $(\mathrm{p}=0,018)$ con una razón de las ventajas para el número de niveles (único/múltiple) de 2,221.

Con el análisis multivariante siguiendo en modelo de regresión lineal considerando edad, sexo y número de niveles intervenidos, observamos que persistía el riesgo de complicaciones del número de espacios intervenidos, independientemente de la edad o el sexo, siendo los múltiples espacios un $117,3 \%$ más frecuente que la intervención de un único (OR 2,173; IC95\% 1,104-4,279).

\section{Discusión}

La columna cervical es el sistema articular más complejo y móvil del organismo. El grado de diferenciación estructural y funcional con que cuenta el raquis cervical es extraordinario, pero el equilibrio de su funcionalidad se ve amenazada de forma constante, incluso en condiciones fisiológicas.

En los últimos 50 años, han sido desarrolladas diversas técnicas de abordaje cervical anterior para tratar una gran variedad de patologías. Este abordaje permite acceder a la duramadre, a las raíces nerviosos, a las arterias vertebrales, y provee de una considerable flexibilidad en el acceso y la extirpación de lesiones, así como la estabilización de la 
columna cervical ${ }^{1}$.

La compresión radicular y de la cara anterior de la médula espinal son las indicaciones más comunes del abordaje cervical anterior. Patologías como el trauma cervical, la herniación aguda del disco, la espondilosis cervical, la osificación del ligamento longitudinal posterior, los procesos neoplásicos o las infecciones, son algunas de las patologías que pueden ser tratadas con éxito mediante esta técnica ${ }^{3}$. Es difícil hoy en día, y a pesar de los muchos trabajos publicados escoger cual es entre los diferentes métodos de fijación o no fijación cervical, el mejor ${ }^{30,48}$.

El Dr. Leroy Abbot fue el primero que sugirió el abordaje cervical anterior en 1952 mientras estaba como profesor invitado en el servicio de los doctores Bailey y Badgley ${ }^{80}$. Este abordaje fue posteriormente utilizado y descrito por estos autores en 19587. En 1958 Robinson y $\mathrm{Smith}^{70}$ introdujeron a la técnica, la utilización del injerto óseo para favorecer la fusión ósea durante la reconstrucción cervical anterior. Cloward ${ }^{13}$ también siguió su técnica, e introdujo el injerto óseo cilíndrico para la fusión cervical anterior. El mayor avance que hubo después se produjo con la instrumentación cervical iniciada por Orozco y Llovet en $1970^{51}$ La tecnología de la fijación cervical se ha desarrollado rápidamente en las últimas tres décadas ${ }^{38}$. Las mejoras técnicas en la instrumentación cervical son muchas veces el resultado de las innovaciones que se han producido en relación a las complicaciones surgidas con los métodos anteriores. Aunque la discectomía por vía anterior es una medida efectiva y generalmente segura ${ }^{4,29,65}$, puede ir asociada a una serie de complicaciones que pueden ser importantes por las estructuras vasculares, respiratorias, neurológicas y digestivas relacionadas. También aunque es considerada una técnica segura y simple, es uno de los procedimientos que más comúnmente recibe denuncias legales ${ }^{29}$.

Una revisión en la literatura revela una extensa lista de complicaciones en la cirugía de la columna cervical por vía anterior $^{9,12,14,22,37,38,39,48,75}$. Si bien, muchas de ellas, son extremadamente $\operatorname{raras}^{38}$.

El porcentaje de complicaciones en la cirugía cervical

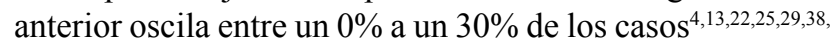
$39,41,49,61,65,69$, un $26 \%$ en nuestro estudio, y un $9.8 \%$ si consideramos sólo como morbilidad las complicaciones que se mantienen de forma permanente.

La perforación de la tráquea, es una rara y poco habitual complicación de este procedimiento, y puede suceder durante la disección medial, en un porcentaje muy inferior al $1 \%{ }^{6}$. Entre un 0,2 a un 3,9\%,,$^{5,38}$ de las discectomías cervicales anteriores se producen complicaciones laríngeas relacionadas con el edema glótico provocado por la retracción laríngea. Ninguno de nuestros pacientes presentaron dichas complicaciones.

En la literatura la disfagia se considera la complicación

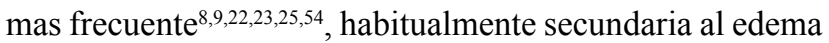
quirúrgico y a la retracción del esófago. El porcentaje de casos de disfagia en nuestro centro fue del 7,7\%. De ellos el $86,6 \%$ de los casos se presentaron de forma transitoria, y se resolvieron en pocos días. En la literatura se habla de alrededor del 5 al $30 \%$ de $\operatorname{casos}^{9,14,15,54}$, de los cuales entre un 0,8 a un $5 \%$ se mantiene de forma permanente. El número de disfagias permanentes encontradas fue del 1,04\%.

La cifra de perforación esofágica se encuentra entre el $0,25 \%$ al $1 \%{ }^{38,39}$, y la perforación faríngea ${ }^{70,75}$ entre el $0,2 \%$ al $1,2 \%$ de las cirugías cervicales anteriores. En nuestra serie ningún paciente presentó estas complicaciones. Esta se produce especialmente en la región cervical alta donde la hipofaringe es delgada. En la mayoría de casos no se detecta durante la cirugía y los síntomas aparecen después como una infección local, fístula, sepsis o mediastinitis $^{14,22,46}$. Muchas veces se produce como consecuencia de la retracción de los tejidos. Otras veces la perforación esofágica se produce por un desplazamiento del injerto.

Tanto el nervio laríngeo superior como el laríngeo inferior (recurrente) se pueden lesionar en el abordaje anterior de la columna cervical. La lesión del nervio laríngeo recurrente representa la complicación que produce más denuncias legales 5 . Los mecanismos por los cuales se produce esta complicación incluyen la presión o estiramiento que originan neuroapraxia, y el edema postoperatorio. La lesión del nervio laríngeo recurrente puede ocasionar disfonía o afonía, fatiga en la voz, tos, y aspiración. Entre un 1\% a un $11 \%,{ }^{11,22,25,30}$, un $3,63 \%$ en nuestro estudio se produce una mínima disfonía después del abordaje anterior. Esta se resuelve sin ningún tratamiento en semanas o meses en la mayoría de los pacientes ${ }^{11,30,45,74}$. La lesión permanente de los nervios laríngeos (1,55\% ) se produce entre el $1 \%$ al $3,5 \%$ en la mayoría de series publicadas ${ }^{11,22,30,45,74}$.

La rotura de la arteria carótida se puede producir por la retracción de los tejidos durante el abordaje anterior ${ }^{7}$. Esta complicación se produce en muy raras ocasiones, pero puede llegar a tener consecuencias devastadoras. Se ha publicado esta complicación en menos de $1 \%$ de los casos. Además es importante considerar que la movilización de la arteria carótida durante la cirugía de la columna cervical puede producir el desprendimiento de una placa de ateroma preexistente y ocasionar un infarto isquémico de origen embólico ${ }^{72}$. No observamos ningún caso con esta complicación, pero se han llegado a comunicar en la literatura más de 100 casos de isquemia cerebrovascular después de la manipulación cervical ${ }^{18,72}$. Al considerar la lesión vascular también tenemos que hablar de la lesión de la arteria vertebral que se produce como consecuencia de la disección agresiva de los músculos largos del cuello ${ }^{8}$, en un $1 \%$ de los casos. Ninguno de nuestros pacientes presentó esta complicación, ni tampoco con lesión en la cadena simpática cervical. Este último se produce entre el $0,2 \%$ al $4 \%$ 
de las cirugías con abordaje anterior ${ }^{22,25,75}$, entre C3 y C6 y se manifiesta por un síndrome de Horner.

La aparición de un déficit neurológico nuevo es una complicación poco frecuente. Si el déficit neurológico se hace evidente inmediatamente después de la intervención la causa mas probable del déficit se debe a un trauma quirúrgico o a problemas relacionados con la posición o la manipulación del cuello durante la intubación ${ }^{8,29}$. Entre un $1,3 \%$ a un $11 \%$ se produce un déficit radicular nuevo, y de un 0,7 a un $3,1 \%$ de empeoramiento del déficit medular ${ }^{22}$. En nuestra serie encontramos un 2,07\% de lesión radicular nueva y un 2,07\% de empeoramiento de la lesión medular. En algunos trabajos hablan del hematoma epidural como complicación de la cirugía cervical anterior. Se produce en un $1 \%$ de las cirugías $8,37,38,39,41,58,61$. No hallamos ningún paciente con ésta complicación.

Entre un $3,1 \%$ a un $14 \%$ se produce la rotura de la duramadre y fístula del líquido cefalorraquídeo. Es una complicación poco frecuente que puede ocurrir durante la exéresis del ligamento longitudinal posterior. No observamos ningún caso de rotura ni fístula de la duramadre. Un paciente presentó un hematoma postoperatorio del lecho quirúrgico $(0,52 \%)$. Se trata de una complicación rara $^{8,14}$, $(1,3 \%)$, y en la mayoría de los casos puede ser tratada de forma conservadora. No por ello debemos despreciarla ya que hematomas importantes que comprometan la vía aérea pueden poner en peligro la vida del paciente a consecuencia de una insuficiencia respiratoria aguda.

La infección postoperatoria puede presentarse en alrededor de un $0,1 \%$ a un $3 \%$ de los pacientes intervenidos ${ }^{6,34}$, $(0,52 \%$ en nuestro centro). Se puede producir en las capas mas superficiales y/o envolver a estructuras profundas. En nuestro caso se produjo por infección superficial de la herida quirúrgica, que fue tratada de forma conservadora con tratamiento antibiótico vía endovenosa. No se produjo ningún caso de infección profunda. En la literatura se cita esta complicación en un $0,9 \%$ de los abordajes cervicales anteriores $^{8}$. Los abscesos epidurales pueden presentarse entre un $0,5 \%$ a un $2 \%$ de $\operatorname{los} \operatorname{casos}^{25,29,38}$ y deben considerar en el diagnóstico diferencial, cuando hay una disfunción neurológica, y signos de sepsis a nivel sistémico.

Las complicaciones relacionadas con el injerto óseo se producen por el colapso, extrusión y migración, y pseudoartrosis. Puede deberse a un tamaño inadecuado del injerto, por trauma postoperatorio, o por movilización inadecuada $^{29}$. El colapso del injerto se produce en un $2 \%$ a un $7 \%$ de las cirugías ${ }^{43}$ (1,55\% en nuestra serie), la mayoría de veces en pacientes ancianos con autoinjerto y con un hueso osteoporótico ${ }^{38}$.

La migración se ha comunicado en un $0,4 \%$ a un $4,6 \%{ }^{6,29,38}$. El desplazamiento del injerto requiere una solución quirúrgica cuando da lugar a disfagia, compromiso respiratorio o déficit neurológico ${ }^{35,50,58}$. En nuestra serie esta complicación se presentó en el 1,03\% de los casos. Un paciente precisó reintervención por disfagia.

La incidencia de complicaciones relacionadas con la instrumentación está entre un 0 a un $33 \%$ de los casos (en un $5,2 \%$ en la presente serie $)^{55}$.

La mortalidad se sitúa entre el $0 \%$ al 1,1\% de los casos, y se producen en la mayoría de ocasiones por complicaciones sistémicas ${ }^{4,26}$. En nuestra serie no tuvimos ningún fallecimiento en el período en que se realizó el seguimiento.

$\mathrm{Ni}$ la edad ni el sexo constituyen un factor de riesgo que determine la presencia de más complicaciones tanto en la bibliografía como en nuestro estudio ${ }^{39,58,59,80}$. Tampoco nosotros hallamos en nuestro estudio esta relación. En un reciente trabajo ${ }^{80}$, donde se estudiaron las complicaciones de 258 pacientes intervenidos de la columna cervical mediante abordaje anterior y se analizaron los posibles factores de riesgo, no se encontró relación estadísticamente significativa.

Tampoco hemos encontrado una relación entre los síntomas iniciales y la presencia de complicaciones ${ }^{58,80,84}$. En algunos trabajos citan un mayor número de infecciones sistémicas en pacientes con mielopatía prequirúrgica ${ }^{68}$, por la mayor inmovilidad, y por la necesidad de sondajes urinarios. En nuestra serie el número de pacientes que presentaron infecciones sistémicas es muy bajo, tan sólo dos pacientes, lo que motiva que no se puedan extraer resultados con significación estadística.

Donde sí hallamos una relación estadísticamente significativa fue en el número de espacios intervenidos, siendo más frecuente las complicaciones en los pacientes tratados de múltiples niveles con una Odds Ratio de 2,173, siendo éste un factor de riesgo de la presencia de más complicaciones, independientemente de la edad o el sexo. En algunas revisiones han analizado el número de niveles intervenidos de forma individualizada y también han hallado más complicaciones, en relación a la instrumentación ${ }^{32,67}$, disfagia en el primer y segundo mes $^{54}$, y algunos casos de un mayor número de migraciones ${ }^{62}$. Por el contrario, otros autores no encuentran una relación estadísticamente significativa entre el número de espacios intervenidos y la presencia de complicaciones ${ }^{21,38,48}$.

En cuanto a las complicaciones en el tipo de cirugía y el material utilizado, hay un importante número de publicaciones en que comparan los distintos sistemas de fijación que se han utilizado en el abordaje anterior con artrodesis ${ }^{48,82,83}$. De todos ellos podemos concluir que no hay un procedimiento ideal que implique un menor número de complicaciones para el tratamiento de la columna cervical por vía anterior ${ }^{48,81,82}$. La mayoría ${ }^{4,16,27,31,36,44,47,60,62,79,81}$ de estudios comparan dos sistemas de fijación y analizan los resultados clínicos, los resultados radiológicos y las complicaciones. En otros trabajos se analizan hasta tres modalidades de tratamiento ${ }^{24,35,76,82}$, pero no hallamos ningún 
estudio que compare todas las modalidades quirúrgicas y métodos de fijación.

Algunos autores comparan la presencia de complicaciones en pacientes con o sin injerto, apreciando más complicaciones en los pacientes en los que se utiliza injerto ${ }^{47}$. En otros estudios se habla de la aparición de un mayor número de complicaciones neurológicas nuevas (lesión medular, lesión radicular) en relación a la estabilidad del segmento $\sin$ injerto óseo ${ }^{11,19,73,77}$. En nuestro estudio no encontramos diferencias estadísticamente significativa en el porcentaje de complicaciones en los pacientes en los que se había utilizado injerto respecto a los que no se había utilizado.

Otros autores comparan las discectomías con injerto con la utilización además de placas y tornillos unicorticales, no encontrando tampoco diferencias estadísticamente significativas en cuanto a las complicaciones ${ }^{27,62,64}$. Nosotros tampoco objetivamos diferencias entre los dos métodos. Al comparar la utilización de cajas intersomáticas con la utilización de injerto, tampoco se hallan diferencias, al igual que en nuestro estudio ${ }^{4,27,35}$. Si comparamos la utilización de cajas intersomáticas más placas con la utilización de cajas intersomáticas solas se describen un menor número de complicaciones cuando se utilizan cajas solas ${ }^{36}$. No encontramos diferencias, en el porcentaje de complicaciones con los distintos métodos, aunque el número de pacientes intervenidos con placa más caja intersomática es bajo.

Cuando comparamos el porcentaje de complicaciones en la cirugía con placas frente a la cirugía con cajas intersomáticas se cita un mayor número de complicaciones con la utilización de placas ${ }^{17}$. En nuestro estudio no hallamos un mayor número de complicaciones en los pacientes en que se había utilizado las placas como método de fijación frente a los otros procedimientos. No hay aún un criterio claro en relación a las complicaciones, pues otros estudios en que se comparan los dos métodos (cajas intersomáticas frente a placas) no encuentran diferencias en el porcentaje de complicaciones en relación al material utilizado ${ }^{24,60}$. Lo que sí podemos decir es que en un reciente trabajo ${ }^{60}$ en que se comparó de forma prospectiva y randomizada, las complicaciones de dos métodos de instrumentación cervical (placas con injerto versus cajas intersomáticas de fibra de carbono) en 40 pacientes, no se hallaron diferencias estadísticamente significativas en las complicaciones que éstos presentaron.

Al analizar los distintos tipos de injertos en la mayoría de trabajos se compara las complicaciones del injerto autólogo, frente al injerto heterólogo ${ }^{4,31,40,53,57,64,66,71}$, y se habla de un mayor porcentaje de pseudoartrosis con el injerto aloinjerto ${ }^{4,34}$, pero también se describen un mayor porcentaje de hematomas de partes blandas, mayor número de infecciones, mayores pérdidas sanguíneas, sin olvidar mayor dolor en el sitio donante, y la presencia de

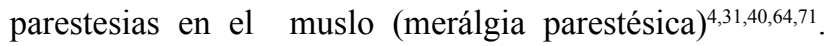
Esta mayor morbilidad que se cita, se resuelven pasado el tiempo, según algunos estudios prospectivos ${ }^{57,66}$. En nuestro trabajo no tuvimos más morbilidad con el injerto autólogo de cresta que con el injerto heterólogo.

El otro injerto utilizado fue el injerto bovino observándose un mayor número de complicaciones en relación a la reabsorción del injerto y la tendencia a la pseudoartrosis de éste frente a los otros dos tipos ${ }^{36,42,56}$, hechos objetivados en nuestro estudio, abandonando su utilización por este motivo.

\section{Conclusiones}

1. La cirugía de la columna cervical por vía anterior, es una técnica simple, y un procedimiento quirúrgicamente seguro con un número bajo de complicaciones.

2. La disfagia es la complicación más frecuente, pero que se encuentra casi inherente al procedimiento y en la mayoría de ocasiones se resuelve sin tratamiento.

3. Han sido muchos los procedimientos utilizados para la fijación de la columna cervical, con más de 40 años de experiencia, y aún queda por definir cual es el mejor. La mayoría de trabajos realizados se basan en estudios de una única institución. Haría falta más estudios de carácter multicéntrico y de cohorte prospectiva para poder comparar resultados clínicos, radiológicos, y la presencia de complicaciones.

\section{Bibliografía}

1. Abraham, D. J., Herkowitz, H.N.: Indications and trends in use in cervical spinal fusions. Orthop Clin North Am 1998; 29: 731-744.

2. Aebi, M., Zuber, K., Marchesi, D.: Treatment of cervical spine injures with anterior plating: Indications, techniques, and results. Spine 1991; 16 (suppl 3): S38-S45.

3. An, H.S.: Clinical presentation of discogenic neck pain, radiculopathy, and myelopathy. En: The Cervical Spine $3^{\text {rd }}$ edition. The Cervical Spine Research Society Editorial Commitee. Philadelphia; Lippincot-Raven 1998; pp. 755.

4. An, H.S., Simpson, J.M., Glover, J.M., Stephany, J.: Comparison between allograft plus demineralized bone matrix versus autograft in anterior cervical fusion. A prospective multicenter study. Spine 1995; 15: 2211-2216

5. Aronson, N.: The management of soft cervical disc protrusions using the Smith-Robinson approach. Clin Neurosurg 1973; 20: 253-258.

6. Aronson, N., Bagan, M., Filtzer, D.L.: Results of using the Smith-Robinson approach for herniated and extruded cervical discs. Technical note. J Neurosurg 1976; 32: 721-722.

7. Bailey, R.W., Badley, C.E.: Stabilization of the cervical spine by anterior fusion. Am J Orthop 1960; 42: 565-594 
(Abstract).

8. Bertalanffy, H., Eggert, H.R.: Complications of anterior cervical discectomy without fusion in 450 consecutive patients. Acta Neurochir 1989; 99: 41-50.

9. Bolhman, H.H.: Cervical myelopathy. En Istructional Course Lectures, American Academy of Orthopaedic Surgeons 1995 (Vol 44).

10. Bryan, V.E.: Cervical motion segment replacement. Eur Spine J 2002; 11 (Suppl 2): S92-97.

11. Bulger, R.F., Rejowski, J.E., Beatty, R.A.: Vocal cord paralysis associated with anterior cervical fusion: considerations for prevention and treatment. J Neurosurg 1985; 62: 657661.

12. Clark, K.: Anterior intraoperative approach for benign extradural cervical lesions. En Youmans JR (ed) Neurological surgery, Vol 3. Philadelphia; Saunders Campany, 1982; p. 2313-2628.

13. Cloward, R.B.: The anterior approach for removal of ruptured cervical disc. J Neurosurg 1958; 15: 602-616.

14. Cloward, R.B. : Complications of anterior disc operation and their treatment. Surgery 1971, 69: 175-182.

15. Connolly, E.S., Seymour, R.J., Adams, J.E.: Clinical evaluation of anterior cervical fusion for degenerative cervical disc disease. J Neurosurg 1965; 23: 431-437.

16. Connolly, P.J., Esses, S.I., Kostuik, J.P.: Anterior cervical fusion: outcome analysis of patients fused with and without anterior cervical plates.J Spinal Disord. 1996; 9:202206.

17. Cho, D.Y., Lee, W.Y., Sheu, P.C.: Treatment of multilevel cervical fusion with cages. Surg Neurol. 2004;62: 378385.

18. Dohn, D.F.: Anterior interbody fusion for treatment of cervical-disk conditions. JAMA 1966; 197: 897-900.

19. Dowd, G.C., Wirth, F.P.: Anterior cervical discectomy: is fusion necessary? J Neurosurg. 1999; 90 (1 Suppl): 8-12.

20. Esperson, J.O., Buhl, M., Eriksen, E.F., Fode, K., Klaerke, A., Kroyer, L., et al.: Treatment af cervical disc disease using Cloward's technique 1. General results, effect of different operative methods and complications in 1106 patients. Acta Neurochir (Wien) 1984; 70: 97-114.

21. Fessler, R.G., Steck, J.C., Giovanini, M.A.:Anterior cervical corpectomy for cervical spondylotic myelopathy. Neurosurgery 1998; 43: 257-265.

22. Flynn, T.B.: Neurologic Complications of Anterior Fusion. Spine 1982; 7: 536-539.

23. Fouyas, I.P., Stonthom, D.F., Sandercock, P.A.: Cochrane rewiew on the role of surgery in spondylotic radiculomyelophaty. Spine 2002; 27: 736-747.

24. Gonzalez-Darder, J.M., Pesudo Martinez, J.V., Feliu Tatay, R.: Post-discectomy cervical fusion. Clinico-radiological study comparing iliac crest bone graft, anterior cervical plate with bone graft and cage-plate. Neurocirugia 2001;12: 143-151.
25. Graham, J.J.: Complications of cervical spine surgery. A five-year report on a survery of the membership of the Cervical Spine Research Society by the Morbidity and Mortality Committee. Spine 1989; 14: 1046-1050.

26. Guidetti, B., Fortuna, A.: Long term results of surgical treatment of myelophaty due to cervical spondylosis. J Neurosurg; 30: 714-721.

27. Hacker, R.J.: A randomised prospective study of an anterior cervical interbody fusion device with a minimum of 2 years of follow-up results. J Neurosurg; 93: 222-226.

28. Hacker, R.J., Cauthen, J.C., Gilbert, T.J., Griffith, S.L.: A prospective randomized multicenter clinical evaluation of an anterior cervical fusion cage. Spine 2000; 25: 2646-2654.

29. Hanbali, F., Gokaslan, Z., Cooper, P.: Ventral and Ventrolateral subaxial descompression. En: Spine Surgery: Techniques, complications, avoidance and management. Philadelphia, Elsevier, 2005; pp. 341-350.

30. Heeneman, H.: Vocal cord paralysis following approaches to the anterior cervical spine. Laryngoscope. 1973; 83: 17-21 (Abstract).

31. Heidecke, V., Rainov, N.G., Marx, T., Burkert, W.: Outcome in Cloward anterior fusion for degenerative cervical spinal disease. Acta Neurochir (Wien) 2000; 142: 283-291.

32. Herkowitz, H.N.: A comparision of anterior cervical fusion, cervical laminectomy, and cervical laminoplasty for the surgical management of multilevel spondylotic radiculopathy. Spine 1998; 13: 774-780.

33. Herkowitz, H.N.: The surgical management of cervical spondylotic radiculopathy and myelopathy. Clin Orthop Relat Res. 1989; 239: 94-108.

34. Hess, T., Gleitz, M., Hanser, U., Mittelmeier, H., Kubale, R.: Primary stability of autologous and heterologous implants for intervertebral body spondylodesis. Z Orthop Ihre Grenzgeb. 1995; 133: 222-226 (Abstract).

35. Kao, F.C., Niu, C.C., Chen, L.H., Lai, P.L., Chen, W.J.: Maintenance of interbody space in one- and two-level anterior cervical interbody fusion: comparison of the effectiveness of autograft, allograft, and cage. Clin Orthop Relat Res. 2005; 430: 108-116.

36. Lofgren, H., Johannsson, V., Olsson, T., Ryd, L., Levander, B.: Rigid fusion after cloward operation for cervical disc disease using autograft, allograft, or xenograft: a randomized study with radiostereometric and clinical follow-up assessment. Spine 2000; 25: 1908-1916.

37. Louis, R.: Surgery of the spine. Springer, Berlin; 1982; pp. 128-135.

38. Lunsford, L.D., Bissonette, D. J., Zorub, D.S.: Anterior surgery for cervical disc disease. Part 1: treatment of lateral cervical disc herniation in 253 cases. J. Neurosurg 1980; 53: $1-11$.

39. Lunsford, L.D., Bissonette, D. J., Zorub, D.S.: Anterior surgery for cervical disc disease. Part 2: treatment of cervical spondylotic myelopathy in 32 cases. J. Neurosurg 1980; 53: 
40. Madawi, A.A., Powell, M., Crockard, H.A.: Biocompatible osteoconductive polymer versus iliac graft. A prospective comparative study for the evaluation of fusion pattern after anterior cervical discectomy. Spine 1996, 21: 2123-2130.

41. Martín, R., Carda J.R, Pinto, J.I, Sanz, F., Montiaga, F., Paternina, B., et al.: Discectomia cervical y artrodesis intersomática mediante la técnica de Cloward: Análisis retrospectivo de las complicaciones y de los resultados radiológicos en 167 casos. Neurocirugía 2002; 13: 265-284.

42. McMurray, G.N.: The evaluation of Kiel bone in spinal fusions. J Bone Joint Surg Br. 1982; 64: 101-104.

43. Modal, C.: Cervical osteochondrosis and disc herniation. Eighteen years' use of interbody fusion by Cloward's technique in 755 cases. Acta Neurochir 1984; 70: 207-225.

44. Moreland, D.B., Asch, H.L., Clabeaux D.E., Castiglia, G.J., Czajka, G.A., Lewis, P.J., et al.: Anterior cervical discectomy and fusion with implantable titanium cage: initialimpressions, patient outcomes and comparison to fusion with allograft. Spine J 2004; 4: 184-191.

45. Netterville, J.L., Koriwchak, M.J., Winkle, M., Courey, M.S., Ossoff, R.H.: Vocal fold paralysis following the anterior approach to the cervical spine. Ann Otol Rhinol Laryngol 1996; 105: 85-91 (Abstract).

46. Newhouse, K.E., Lindsey, R.W., Clark, C.R., Lieponis, J., Murphy, M.J.: Esophageal perforation following anterior cervical spine surgery. Spine 1989; 14: 1051-1053.

47. Nohra, G., Abi-Lahoud, G., Jabbour, P., Salloum, C., Rizk, T., Samaha, E., et al.: Anterior cervical discectomy with or without bone graft placement in the treatment of cervical radiculopathy. Long-term results. Neurochirurgie 2003; 49:571-578.

48. Nirala, A.P., Husain, M., Vatsal, D.K.: A retrospective study of multiple interbody grafting and long segment strut grafting following multilevel anterior cervical decompression. Br J Neurosurg 2004; 18: 227-232.

49. Oga, M., Yuge, I., Terada, K., Shimizu, A.: Tortuosity of vertebral artery in patients with cervical spondylotic myelophaty. Risk factors for vertebral artery injury durins anterior cervical descompresion. Spine 1996; 21: 1085-1089.

50. Omeis, I., DeMattia, J.A., Hillard, V.H., Murali, R., Das, K.: History of instrumentation for stabilization of the subaxial cervical spine. Neurosurg Focus 2004; 16: E10: 120.

51. Orozco-Declos, R., Llovet-Tapies, J.: Osteosintesis en las fracturas del raquis cervical: nota técnica. Revista de Ortopedia y Traumatología 1970; 14: 285-288.

52. Payer M, May D, Reverdin A,,Tessitore E: Implantation of an empty carbon fibre composite frame cage after single-level anterior cervical discectomy in the treatment of cervical disc herniation: preliminary results. J Neurosurg 2003; 98 (Suppl 2): 143-148.

53. Pitzen, T., Kranzlein, K., Steudel, W.I., Strowitzki, M.:
Complaints and findings at the iliac crest donor site following anterior cervical fusion. Zentralbl Neurochir 2004; 65: 7-12 (Abstract).

54. Rajesh, B.; Lee, M. J., Yoo, J.U.: Incidence of Dysphagia After Anterior Cervical Spine Surgery: A Prospective Study. Spine 2002 Nov; 27): 2453-2458.

55. Rappaport, L.H., O’Leary, P.F.: Cervical disc disease. En: Bridwell KH, Dewald RL (eds): Textbook of Spinal Srgery. Philadelphia, Lippincott-Raven, 1997, pp 1371-1396.

56. Rawlinson, J.N.: Morbidity after anterior cervical decompression and fusion. The influence of the donor site on recovery, and the results of a trial of surgibone compared to autologous bone. Acta Neurochir (Wien) 1994; 131: 106-118.

57. Robertson, P.A., Wray, A.C.: Natural history of posterior iliac crest bone graft donation for spinal surgery: a prospective analysis of morbidity. Spine 2001; 26: 1473-1476.

58. Romano, P.S., Campo, D.R., Rainwater, J.A.: Elective cervical discectomy in California: postoperative in-hospital complications and their risk factors. Spine 1997; 22: 26772692.

59. Rozack, N., Greenberg, J., Green, B.A.: Surgery for cervical mielophaty in geriatric patients. Spinal Cord 1998; 36: 629-632

60. Ryu, S.I., Mitchell, M., Kim, D.H.: A prospective randomized study comparing a cervical carbon fiber cage to the Smith-Robinson technique with allograft and plating: up to 24 months follow-up. Eur Spine J 2005; Jun 25.

61. Salcman, M.: Complications of cervical spine surgery. Crit Care Med 2000;. 29: 2027-2028.

62. Samartzis, D., Shen, F.H., Lyon, C., Phillips, M., Goldberg, E.J., An, H.S.: Does rigid instrumentation increase the fusion rate in one-level anterior cervical discectomy and fusion? Spine J 2004; 4: 636-643.

63. Saveland, H., Aspenberg, P., Zygmunt, S., Herrlin, K., Christensson, D., Rydholm, U.: Bovine bone grafting in occipito-cervical fusion for atlanto-axial instability in rheumatoid arthritis. Acta Neurochir (Wien) 1994; 127: 186-190.

64. Savolainen, S., Usenius, J.P., Hernesniemi, J.: Iliac crest versus artificial bone grafts in 250 cervical fusions. Acta Neurochir (Wien) 1994; 129: 54-57.

65. Schmidek, H. H., Smith, D. A.: Anterior cervical disc excision in cervical spondylosis. En: Schmidek HH, Sweet WH (eds): Operative Neurosurgical Techniques, ed 3, vol 2. Philadlephia, WB Saunders, 1995, pp. 1783-1804.

66. Schnee, C.L., Freese, A., Weil, R.J., Marcotte, P.J.: Analysis of harvest morbidity and radiographic outcome using autograft for anterior cervical fusion. Spine 1997; 22: 22222227.

67. Schultz, K.D. Jr, McLaughlin, M.R., Haid, R.W. Jr, Comey, C.H., Rodts, G.E. Jr, Alexander, et al.J.: Single-stage anterior-posterior decompression and stabilization for complex cervical spine disorders. J Neurosurg 2000; 93 (2 Suppl): 214-218. 
68. Shaffrey, M.E., Sholes, A.H.,: Medical complications. En Benzel EC (ed.) Spine Surgery. Techniques, complications avoidance and management. New York, Churchill Livingstone, 1999. 1459-1463.

69. Shinomiya, K., Okamoto, A., Kamikozuru, M., Furuya, K., Yamura, J.: An analysis of failures in primary cervical anterior spinal cord decompression and fusion. J Spinal Disor 1993; 6: 277-288.

70. Smith, G.W., Robinson, R.A.: The treatment of certain cervical-spine disorders by anterior removal of the intervertebral disc and interbody fusion. J Bone Joint Surg Br (Am) 1958; 40: 607-624.

71. Silber, J.S., Anderson, D.G., Daffner, S.D., Brislin, B.T., Leland, J.M., Hilibrand, A.S., et al.: Donor site morbidity after anterior iliac crest bone harvest for single-level anterior cervical discectomy and fusion. Spine 2003; 28 : 134-139.

72. Silber, J.S., Haldeman, S., Kohlbeck, F.J., McGregor, M.: Unpredictability of cerebrovascular ischemia associated with cervical spine manipulation therapy. Spine 2002; 27: 4955.

73. Sonntag, V.K., Klara, P.: Controversy in spine care. Is fusion necessary after anterior cervical discectomy? Spine 1996; 21: 1111-1113.

74. Sperry, R.J., Johnson, J.O., Apfelbaum, R.I.: Endotracheal tube cuff pressure increases significantly during anterior cervical fusion with the Caspar instrumentation system. Anesth Analg 1993; 76: 1318-1321.

75. Tew, J.M., Mayfield, F.H. : Complications surgery of the anterior cervical spine. Clin Neurosurg; 23: 424-34.

76. Thalgott, J.S., Xiongsheng, C., Giuffre, J.M.:. Single stage anterior cervical reconstruction with titanium mesh cages, local bone graft, and anterior plating. Spine J 2003; 3 : 294-300.

77. Thorell, W., Cooper, J., Hellbusch, L., Leibrock, L.: The long-term clinical outcome of patients undergoing ante-

\section{Comentario al trabajo: Complicaciones de la discectomía cervical anterior: estudio retrospectivo en 193 pacientes de $R$. García-Armengol y cols.}

El trabajo realizado por R. García-Armengol y cols. destacaría el seguimiento de una amplia serie (193 pacientes) intervenidos exclusivamente por dos cirujanos, los cuales nos muestran su experiencia y nos hacen referencia a las complicaciones que han observado en el abordaje anterior de la patología cervical, que realmente han sido escasas. Es necesario destacar, según mencionan los autores, que éstas aumentan en relación con los niveles intervenidos.

Por otra parte, en la amplia revisión que realizan en la literatura describen en la discusión que no hay que olvidar que independientemente del empeoramiento neurológico que se puede dar, también se pueden observar la afectación rior cervical discectomy with and without intervertebral bone graft placement. Neurosurgery 1998; 43: 268-273.

78. Vavruch, L., Hedlund, R., Javid, D., Leszniewski, W, Shalabi, A.: A prospective randomized comparison between the cloward procedure and a carbon fiber cage in the cervical spine: a clinical and radiologic study. Spine 2002; 27: 16941701.

79. Wang, J.C., Hart, R.A., Emery, S.E., Bohlman, H.H.: Graft migration or displacement after multilevel cervical corpectomy and strut grafting. Spine 2003; 28: 1016-1021.

80. Wang, J.C., Liang, F.G., Qu, D.B., Jin, D.D.: Risk factors analysis of early complications following anterior spine surgery in 258 cases of cervical spondylotic myelophaty. Di Yi Jun Yi Do Xue Bao 2005; 25: 450-453 (Abstract).

81. Watters, J., Levinthal, R.: Anterior cervical discectomy with and without fusion. Results, complications, and longterm follow-up. Spine 1994; 19: 2343-2347.

82. Wirth, F.P., Dowd, G.C., Sanders, H.F., Wirth, C.: Cervical discectomy. A prospective analysis of three operative techniques. Surg Neurol 2000; 53: 340-346.

83. Zaveri, G.R., Ford, M.: Cervical spondilosis: the role of anterior instrumentation after decompression and fusion. Jspinal Disord 2001; 14: 10-16.

84. Zeidman, S.M., Ducker, T.B., Raycraft, J.: Trends and complications in cervical spine surgery:1989-1993. J Spinal Disord. 1997; 10: 523-526.

Garcia-Armengol, R.; Colet-Esquerre, S.; Teixidor-Rodríguez, P.; Alamar-Abril, M.; Cladellas-Ponsa, J.M.; Hostalot-Panisello, C.; Muñoz-Aguiar, J.; Florensa-Brichs, R.: Complicaciones del abordaje anterior en la patología de la columna cervical. Neurocirugía 2007; 18: 209-220.

Correspondencia postal: Roser Garcia Armengol. Servicio de Neurocirugía. Hospital Universitari Germans Trias i Pujol. Carretera de Canyet s/n. 08916 Badalona (Barcelona).

de estructuras como la traquea y el esófago que pueden provocar fístulas y en algunos casos medioastinitis, o la afectación de los grandes vasos como son la arteria carótida y la arteria vertebral. Con respecto a las complicaciones de hematomas postquirúrgicos residuales, que los autores describen en un caso de la serie, aconsejaría siempre la colocación de drenaje en el lecho operatorio que probablemente lo realizan sistemáticamente aunque no lo hayan mencionado en el texto. En definitiva es un trabajo interesante y que aporta una buena revisión de la literatura.

A. Isla Madrid 